Interfacing external sensors with android smartphones through near field communication

This content has been downloaded from IOPscience. Please scroll down to see the full text.

Download details:

IP Address: 129.27.173.177

This content was downloaded on 09/01/2017 at 17:56

Manuscript version: Accepted Manuscript

Leikanger et al

To cite this article before publication: Leikanger et al, 2017, Meas. Sci. Technol., at press: http://dx.doi.org/10.1088/1361-6501/aa57da

This Accepted Manuscript is copyright Copyright 2017 IOP Publishing Ltd

During the embargo period (the 12 month period from the publication of the Version of Record of this article), the Accepted Manuscript is fully protected by copyright and cannot be reused or reposted elsewhere.

As the Version of Record of this article is going to be / has been published on a subscription basis, this Accepted Manuscript is available for reuse under a CC BY-NC-ND 3.0 licence after a 12 month embargo period.

After the embargo period, everyone is permitted to use all or part of the original content in this article for non-commercial purposes, provided that they adhere to all the terms of the licence https://creativecommons.org/licences/by-nc-nd/3.0

Although reasonable endeavours have been taken to obtain all necessary permissions from third parties to include their copyrighted content within this article, their full citation and copyright line may not be present in this Accepted Manuscript version. Before using any content from this article, please refer to the Version of Record on IOPscience once published for full citation and copyright details, as permissions will likely be required. All third party content is fully copyright protected, unless specifically stated otherwise in the figure caption in the Version of Record.

When available, you can view the Version of Record for this article at: http://iopscience.iop.org/article/10.1088/1361-6501/aa57da 


\section{Interfacing External Sensors with Android Smartphones Through Near Field Communication}

Tore Leikanger, Juha Häkkinen and Christian Schuss

Circuits and Systems (CAS), University of Oulu, Erkki Koiso-Kanttilan katu 3, 90570 Oulu, Finland

E-mail: tore.leikanger@oulu.fi

December 2016

Abstract. In this paper, we present and evaluate a new approach to communicate with inter-integrated circuit (I2C) enabled circuits such as sensors over near field communication (NFC). The NFC-to-I2C interface was designed using a non-standard NFC command to control the $\mathrm{I} 2 \mathrm{C}$ bus directly from a smartphone, which was controlling both, the read and write operations on the I2C bus. The NFC-to-I2C interface was reporting back the data bytes on the bus to the smartphone when the transaction was completed successfully. The proposed system was tested experimentally, both, with write and read requests to a commercial microcontroller featuring a hardware $\mathrm{I} 2 \mathrm{C}$ port, as well as reading a commercial $\mathrm{I} 2 \mathrm{C}$ enabled humidity and temperature sensor. We present experimental results of the system which show that our approach enables an easy interface between smartphones and external sensors. Interfacing external sensors is useful and beneficial for smartphone users, especially, if certain types of sensors are not available on smartphones.

Keywords: embedded systems, inter-integrated circuit, near field communication, NFC interface, protocols, sensors, wireless interface. Submitted to: Meas. Sci. Technol. 


\section{Introduction}

Near Field Communication (NFC) is an increasingly popular technology, which lately became a common feature in most of the medium and high-end Android smartphones. In smartphones, the technology is most used for Bluetooth pairing to share data between devices. Recently, NFC payment systems have been developed which can be embedded in credit cards or accessed using smartphone applications. Furthermore, NFC tags can be used for e.g. smart poster applications and for ticketing systems [1,2].

Recent research has been exploring the possibility of using NFC as a measurement system. The NFC enabled sensors are usually dual-interface devices, embedding an NFC proximity inductive coupling card (PICC) core at one end and an inter-integrated circuit (I2C) slave core on the other [3, 4]. Another approach is to emulate the NFC PICC with an NFC proximity coupling device (PCD) circuit, and then control the circuit through a microcontroller at the sensor side [5]. Both of these approaches have the draw-back of requiring an active master device on the sensor side for controlling both the sensor and the NFC circuit.

In [6], the NFC frequency is used for temperature measurements, but not the NFC digital protocol itself. Thus, this approach cannot be used to read temperature sensors with the help of smartphones or other devices through NFC. The use of a NFC sensor platform with commercial components reduces the flexibility for possible modifications to the system [7]. Reading sensors through NFC can be useful in various different types of applications, for example, in medical applications for obtaining information from blood sugar sensors $[8,9,10]$.

The proposed system in this paper can be useful for obtaining data wirelessly from different types of embedded sensors which can be integrated, for example into bicycles, and wearable sensors which are placed on top of the human body $[11,12]$. The aim of this research is not development of new sensor technologies. The main focus lies on wireless data collection and using the smartphone for the visualisation of the collected data. At present, this type of data is often collected by a data acquisition (DAQ) module and then visualised on a computer [13].

In this paper, we present a new approach of using the NFC circuit as a bridge between a I2C slave and a smartphone acting as the $\mathrm{I} 2 \mathrm{C}$ master. This method removes the need of an active master device between the NFC circuit and I2C slave circuits such as sensors. This approach also allows more flexible solutions. For example, with the help of our system, firmware updates are simplified and can be done purely in the smartphone software.

The proposed system was experimentally verified and the experimental results are presented in this paper. The verification of the data transaction was done in two ways. Firstly, sensor readings were verified with the help of a commercial microcontroller which featured a hardware I2C port. Secondly, sensor information was read through a field-programmable gate array (FPGA) development platform on which the NFC-to-I2C interface was implemented. The results of both tests are compared with each other to verify if the data transmission through the NFC-to-I2C interface was successful.

Analyzing indoor environment conditions can be one of the possible application fields for the developed NFC-to-I2C interface. Here, in a typical use case scenario, a mobile application on a smartphone can be used for uploading measurement data to a cloud service. Monitoring and evaluating ambient conditions such as the humidity and temperature levels can be helpful to provide users feedback on the indoor air quality (IAQ) of their living and working environments $[14,15,16]$. Similarly, the air quality inside and outside a car can be analysed with the help of different air sensor modules [17].

The structure of this paper is as follows: First, the similarities and differences between the NFC protocol and the $\mathrm{I} 2 \mathrm{C}$ protocol are elaborated as well as the opportunities for controlling the I2C bus over NFC. Secondly, the configuration of the implemented NFC core and the $\mathrm{I} 2 \mathrm{C}$ core are described and discussed. Then, the experimental set-up is described and the experimental results are presented. Finally, the results are summarized and the paper is concluded.

\section{NFC and I2C protocols}

The NFC and I2C protocols are similar in many aspects. Both protocols have a very strong masterslave relationship, in which the master always controls the bus and the slave only responds to the master commands. NFC slaves (PICCs) only respond when they are in their active state, while $\mathrm{I} 2 \mathrm{C}$ slaves only respond if the command includes the correct address. 
This feature enables multiple slave buses for both technologies.

However, NFC is a single master multiple slave technology, while $\mathrm{I} 2 \mathrm{C}$ is a multiple master multiple slave technology. In addition, I2C devices can switch between being a master and a slave on the $\mathrm{I} 2 \mathrm{C}$ bus, which is not possible for NFC devices with the exception of the peer-to-peer mode (a dual master no slave system) [18, 19].

\subsection{The used NFC protocol}

The PICC core which is used in the proposed system is a NFC Forum type 2 tag and a subgroup of the NFCA standard [20]. The PICC core features 7 unique identification (UID) bytes and 48 user memory bytes, and all standard NFC Forum commands for type 2 tags including read and write capability.

NFC-A, as well as other NFC technologies, is a short-range radio-frequency technology using an amplitude modulated $13.56 \mathrm{MHz}$ electromagnetic field for communication. The range of NFC technologies is up to $10 \mathrm{~cm}$, typically a couple of centimeters for NFC readers in smartphones [4]. PCD to PICC (reader to tag) communication is carried out with modified Miller encoding, and PICC to PCD communication is established with Manchester encoding on an $848 \mathrm{kHz}$ sub-carrier. The bit rate for NFC Forum type 2 tags is $106 \mathrm{kHz}$ [21].

\subsection{The I2C protocol}

Basically, the $\mathrm{I} 2 \mathrm{C}$ protocol is a two wire communication protocol in which the data transfer is synchronized on the clock line (SCL), and the data is transferred on the data line (SDA). For standard low-speed I2C systems (up to $400 \mathrm{kHz}$ ), the SCL and SDA lines are pulled up using $4.7 \mathrm{k} \Omega$ resistors, and the clocking of the bus as well as the data transfer is done by actively driving the lines to ground [19].

The $\mathrm{I} 2 \mathrm{C}$ anti-collision is achieved by sending a start condition to the $\mathrm{I} 2 \mathrm{C}$ bus. Hereby, the first one sending a start condition obtains control over the bus until a stop condition is sent. If another master attempts to use the bus in the meantime, this master has to wait until the bus is again free [19]. When the master is sending data over the $\mathrm{I} 2 \mathrm{C}$ bus, the master has to first send the $\mathrm{I} 2 \mathrm{C}$ slave address (usually 7 bits) and RW flag indicating a read or a write request. Reading data over the $\mathrm{I} 2 \mathrm{C}$ bus is done by first writing a command to the $\mathrm{I} 2 \mathrm{C}$ slave, and then reading the requested data [19].

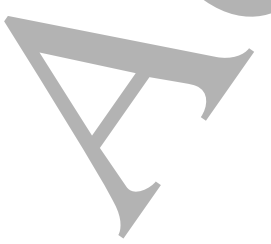

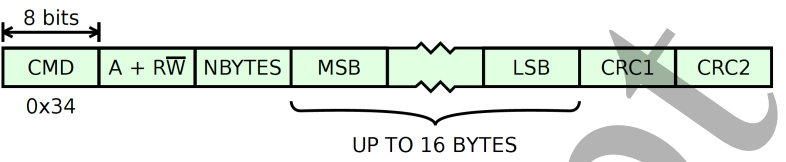

Figure 1: The NFC I2C request used to initiate the I2C serial communication. CMD is the command code, $\mathrm{A}+\mathrm{R} \bar{W}$ is the 7-bit address and the RW flag, NBYTES is the number of bytes, MSB is most significant byte, LSB is the least significant byte, and CRC1 and 2 are the cyclic redundancy check bytes.

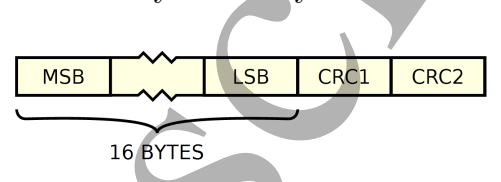

Figure 2: Response to the NFC-I2C request. MSB is most significant byte, LSB is the least significant byte, and $\mathrm{CRC} 1$ and 2 are the cyclic redundancy check bytes.

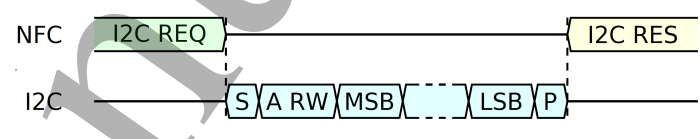

Figure 3: Timing of the NFC-I2C request and response, and the $\mathrm{I} 2 \mathrm{C}$ transmission. $\mathrm{S}$ is the start condition, MSB is most significant byte, LSB is the least significant byte, and $\mathrm{P}$ is the stop condition

Table 1: Description of the $\mathrm{NFC}-\mathrm{I} 2 \mathrm{C}$ request and response

\begin{tabular}{ccc}
\hline Byte & I2C request & I2C response \\
\hline CMD & Command code & \\
A + RW & Address and RW flag & \\
NBYTES & Number of bytes & \\
MSB & MSB of data & MSB of data \\
LSB & LSB of data & LSB of data \\
CRC1\&2 & CRC bytes & CRC bytes \\
\hline
\end{tabular}

\subsection{Controlling the I2C bus over $N F C$}

A non-standard command has been implemented in the NFC Forum type 2 core described above, exclusively to control the I2C bus. This command includes the I2C address, the RW flag, as well as the number of bytes to read or write over the $\mathrm{I} 2 \mathrm{C}$ bus, before the actual data bytes can be transmitted. The PICC then responds with the actual bytes on the $\mathrm{I} 2 \mathrm{C}$ bus, used to verify the data written or to return the data read over the $\mathrm{I} 2 \mathrm{C}$ bus. The non-standard NFC $\mathrm{I} 2 \mathrm{C}$ request is shown in figure 1, the NFC-I2C response is shown in figure 2, and the timing is shown in figure 3. Table 1 presents a description of each byte in the NFC-I2C request and response. 


\section{The digital design of the NFC-I2C interface}

\subsection{The NFC core}

The NFC core is based on the NFC Forum type 2 PICC described in the NFC Forum standard documents $[20,21]$. For this tag to work, several sub-modules are needed including a modified Miller demodulator, a CRC generator, request and response logic, as well as a Manchester code modulator [21]. The sub-modules are connected as shown in figure 4 . Furthermore, as we are dealing with type 2 PICC with 7 UID bytes, we need to design the core around a finite state machine with the states IDLE, READY 1, READY 2, ACTIVE and HALT.

The IDLE state is the initial state, i.e. the state which the NFC core enters during a reset or a power on. The READY 1 and 2 states are the handshake/anticollision states used by the PCD to initialise the PICC. The ACTIVE state is the main state of the core, in which the actual data transfer between the PCD and PICC is realized, such as reading from the memory on the one hand and writing to the memory on the other. The HALT state is used for putting the PICC to an inactive state while the PCD is communicating with other PICCs, for the case that other PICCs are present in the field [20].

The demodulator is regenerating the data bits and is shifting them into a data byte, before forwarding the byte to the request and response logic. When the request is completely sent, i.e. all request bytes including the CRC bytes are received, they are shifted into the CRC generator to confirm that the request is free of errors. The request and response logic then continues to interpret the request.

Now, if the request is valid, the request and response logic must decide whether the PICC should respond or not depending on the state of the PICC. More details about the requests to which a PICC is responding and more information on the possible states of a PICC can be found in the NFC Forum standard documents $[20,21]$. In addition to the functionality which is described in the NFC Forum documents, the $\mathrm{I} 2 \mathrm{C}$ request is added into the NFC core, as described above. If a response needs to be sent to the PCD, the response is shifted into the Manchester code modulator.

The NFC-I2C request becomes available when the PICC enters the ACTIVE state. When the request is received, the $\mathrm{NFC}$ core initiates an $\mathrm{I} 2 \mathrm{C}$ transfer (read or write) in the $\mathrm{I} 2 \mathrm{C}$ core, and then responds to the NFC-I2C request and transfers data bytes accordingly. The connection between the NFC core and the I2C core is shown in figure 4.

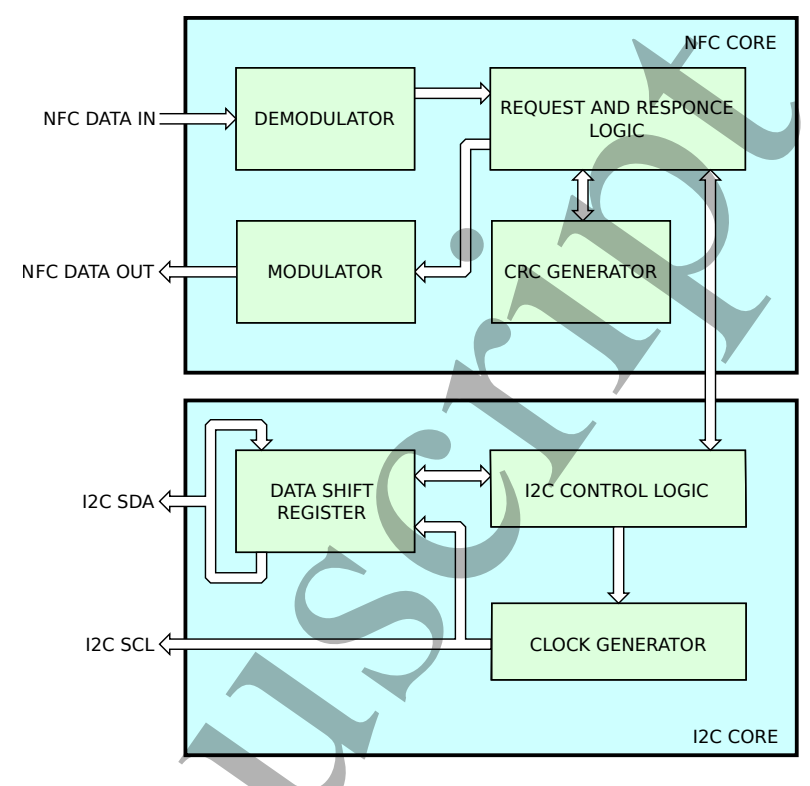

Figure 4: The NFC core and the $\mathrm{I} 2 \mathrm{C}$ core, showing the connection between the two cores.

\subsection{The I2C core}

The I2C core features a shift register for shifting in and out data through the SDA line. Furthermore, a clock generator is embedded in the core for controlling the time and pace of the data shifting and for generating the clock signal on the SCL line. The shift register was slightly modified in order to send a start signal at the beginning of a data transfer and a stop signal at the end of the data transfer. In addition, the modification allowed us as to send an acknowledgement (ACK) signal as well as a negative acknowledgement (NAK) signal between data bytes when the I2C core is reading over $\mathrm{I} 2 \mathrm{C}$.

Both, the SDA and the SCL line are dual direction lines, with internal pull-down transistors and external pull-up resistors. The data lines allow data transfer in both directions and also allow the I2C slave to delay the data transfer by pulling the SCL line down. The connections between the different parts of the $\mathrm{I} 2 \mathrm{C}$ core are shown in figure 4.

The data shift register, as shown in figure 4, latches data from the $\mathrm{I} 2 \mathrm{C}$ logic when requested, and shifts out this data on every falling edge on the SCL line. Moreover, the data on the SDA line is shifted into this shift register on every rising edge on the SCL line. It is worth noting that data in the shift register is stationary when the SCL line is pulled low by a I2C slave device. Between each byte, i.e. during the ACK/NAK time, the data in the shift register is made available to the $\mathrm{I} 2 \mathrm{C}$ control logic while the next byte is latched in from the $\mathrm{I} 2 \mathrm{C}$ control logic. 


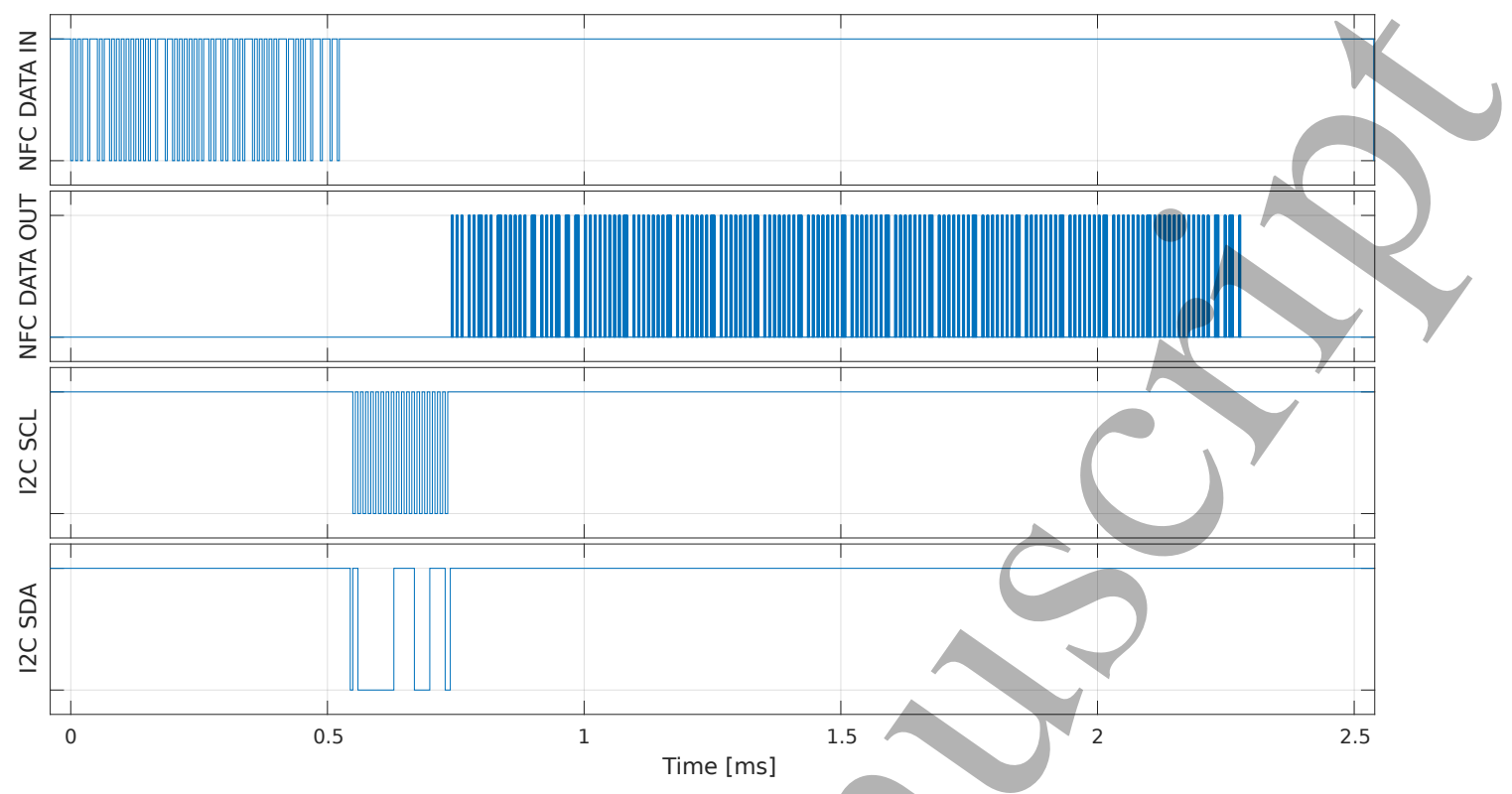

Figure 5: The waveforms at the NFC and I2C pins during an NFC-I2C request and response as measured by a logic analyzer.

3.3. Waveforms at the inputs and outputs of the NFC and I2C cores

The waveforms at the $\mathrm{NFC}$ and $\mathrm{I} 2 \mathrm{C}$ pins during an $\mathrm{NFC-I2C}$ request and response were measured by a logic analyzer and are shown in figure 5. In this example waveform, the NFC-to-I2C interface requests to write a data byte with value $0 x \mathrm{xE} 3$ to an $\mathrm{I} 2 \mathrm{C}$ slave with address $0 x 40$ by the Samsung Galaxy A3 smartphone. This is the same command to initiate a temperature measurement with the SHT21 humidity and temperature sensor [25].

As can be seen in figure 5 , the $\mathrm{I} 2 \mathrm{C}$ core initiates the $\mathrm{I} 2 \mathrm{C}$ communication at once when the NFC-I2C request has been received, and initiates the NFC-I2C response immediately after the $\mathrm{I} 2 \mathrm{C}$ communication is completed. Because the maximum number of bytes in the $\mathrm{I} 2 \mathrm{C}$ communication has been set to 16 , in this NFC-to-I2C interface, the time between the NFC-I2C request and response is within the limits in the NFC Forum standard [21].

\subsection{Sources of error in the NFC-to-I2C interface}

NFC data is hard to corrupt due to its data integrity checks, including both cyclic redundancy check (CRC) and parity bits. Also, NFC modulation is hard to modify by a man-in-the-middle attack, because of the used modulation scheme [21]. The I2C protocol standard, however, does not include data integrity checks [22]. Of course, it is possible to include CRC if the slave circuits supports this feature. Since the
NFC data is hard to corrupt, one possible source of error is the implementation of the $\mathrm{I} 2 \mathrm{C}$ master and the I2C bus, as well as the data transfer between the I2C master and the NFC core.

\section{Experimental verification of the proposed system}

\subsection{Experimental set-up}

For the implementation of the NFC forum type 2 core, we chose an FPGA development platform with specific features for NFC development such as a NFC analog front end (influenced by the Chameleon mini project [23]), a power circuitry, an electrically erasable programmable read only memory (EEPROM), and an Altera Max 10 FPGA. The development platform also features 16 general purpose in-out pins available on the circuit board on which $\mathrm{I} 2 \mathrm{C}$ bus is included. Figures 6 and 7 show a block diagram and a picture of the designed device.

In this paper, the presented design works as a prototyping platform for an application specific integrated circuit (ASIC) which features the NFC-toI2C interface. The current requirement of an external power supply can be a limiting factor in certain application scenarios. It is worth noting, that the presented system in this paper includes components for development purposes which are not needed in a design for commercial applications. Furthermore, we also designed an energy harvesting circuit so that the ASIC works as a batteryless passive NFC tag. The 


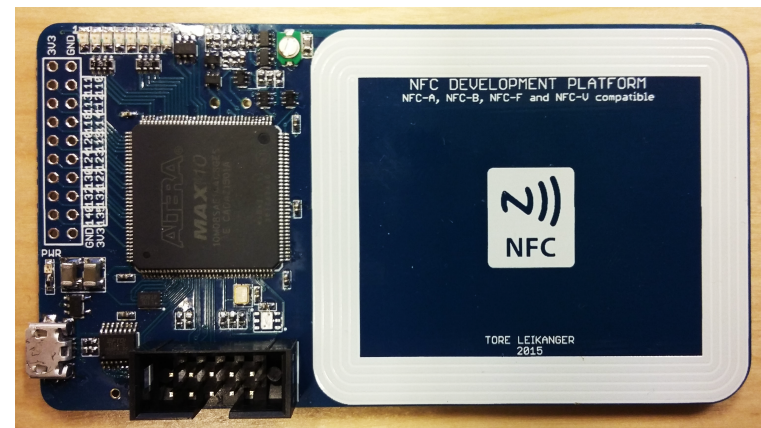

(a) Picture of the FPGA based NFC development device.

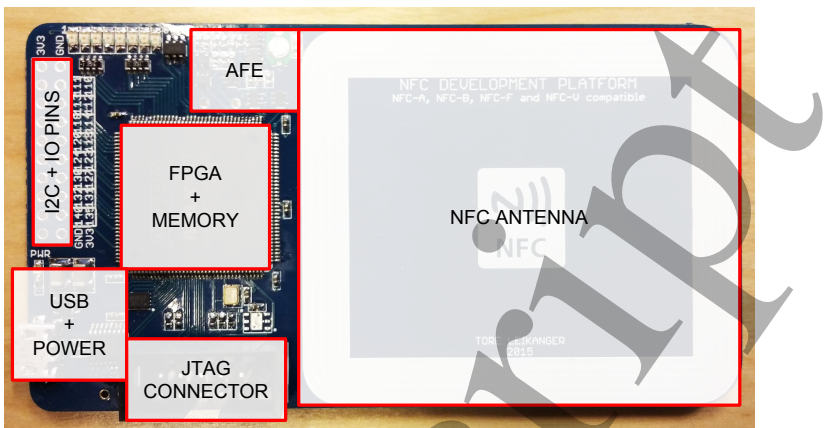

(b) Description of the different parts of the NFC development device.

Figure 6: Picture showing the NFC development device with (b) and without (a) descriptions.

NFC-to-I2C interface as well as the sensors will then be powered by the smartphone.

The experimental set-up can be seen in figure 8 . The multiple master feature on the $\mathrm{I} 2 \mathrm{C}$ bus allows to read the same sensor using the NFC-to-I2C interface and the microcontroller at the same time. This feature allows minimizing any error which can be caused due to a delay between measurements.

The microcontroller is programmed to work as both as a I2C master and as a I2C slave. This type of configuration requires two separate tests which need to be carried out. The first test is the echo test, in which the NFC-to-I2C interface transmits a data byte to the microcontroller before the NFC-to-I2C interface reads the same data byte from the microcontroller. This test is then successful if the data byte remains the same during the whole test period.

The second test requires the microcontroller to read the sensor each time the sensor is read by the NFC-to-I2C interface. Here, the microcontroller also reports the data to a host computer through an USB connection. The microcontroller chosen for this application is the Atmel Atmega328P used in the Arduino platform [24], and the senson is the Sensirion SHT21 humidity and temperature sensor [25]. The smartphone used to perform the tests is the Samsung Galaxy A3.

The Android programming code needed for implementing the NFC-I2C request is provided below. This example code initiates a temperature measurement on the Sensirion SHT21 humidity and temperature sensor, and then requests reading the current temperature.

byte [] init_temp $=\{$ (byte) $0 \times 34$, (byte) $0 \times 80$, (byte) $0 \times 01$, (byte) $0 \times E 3\}$;

byte [] read_data $=\{$ (byte) $0 \times 34$, (byte) $0 \times 81$, (byte) 0x03\};

tag.transceive (init_temp);

byte [] data = tag.transceive (read_data);

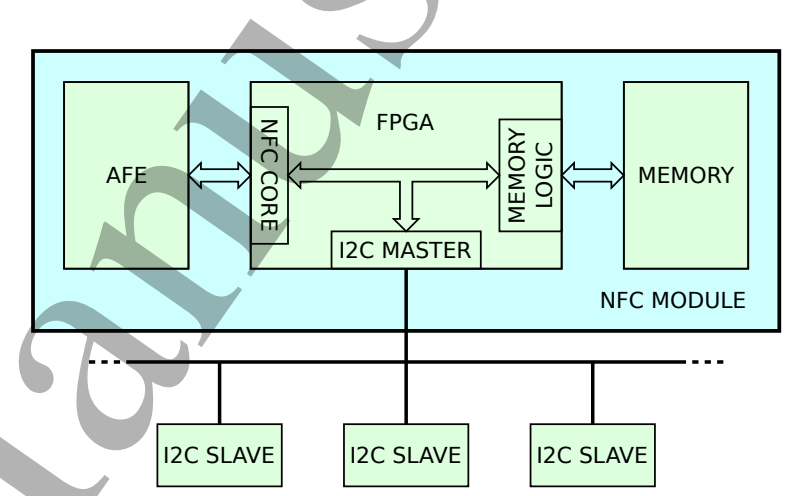

Figure 7: The different blocks on the NFC tag module. AFE is the analog front end.

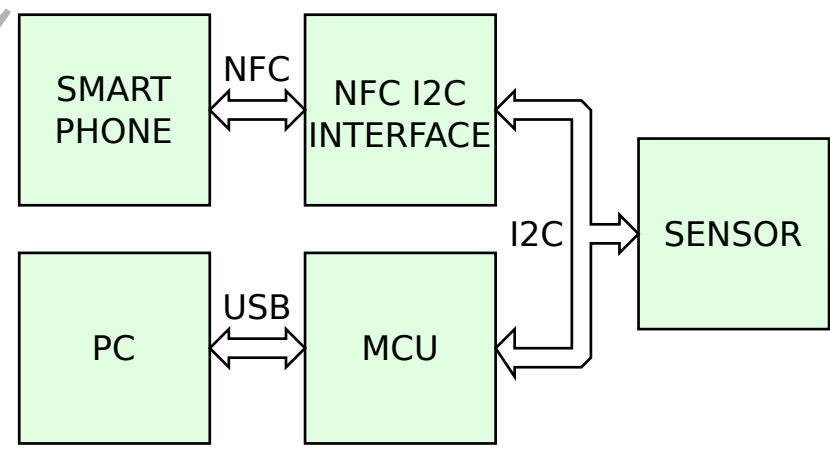

Figure 8: Experimental set-up for verifying the functionality of the circuit. MCU is the microcontroller.

The raw received data is then converted to degrees Celsius as instructed by the datasheet [25] by the following commands:

double raw_temp $=(\operatorname{data}[0] \& 0 \mathrm{xFF}) / 256$.
$+(\operatorname{data}[1] \& 0 \mathrm{xFC}) / 65536$.

double temp $=-46.85+(175.72 *$ raw_temp $)$;

To read the humidity, init_humid $=\{0 \times 34,0 \times 80,0 x 01$, $0 x E 5\}$ was used [25]. 
Table 2: I2C echo test. A random data byte is sent to the microcontroller, and then read by the phone. The phone reports the value of both the sent and the received data byte, and the microcontroller reports the received data byte.

\begin{tabular}{|c|c|c|}
\hline Sent (phone) & Received (phone) & Reported (MCU) \\
\hline $0 \times 3 \mathrm{~A}$ & $0 \times 3 A$ & $0 \times 3 A$ \\
\hline 0xE6 & 0xE6 & 0xE6 \\
\hline $0 \mathrm{xCD}$ & $0 \mathrm{xCD}$ & $0 \mathrm{xCD}$ \\
\hline $0 \times 84$ & 0x84 & $0 \times 84$ \\
\hline $0 \mathrm{xC} 2$ & $0 \mathrm{xC} 2$ & $0 \mathrm{xC} 2$ \\
\hline $0 \times 20$ & $0 \times 20$ & $0 \times 20$ \\
\hline $0 \times 49$ & $0 \times 49$ & $0 \times 49$ \\
\hline $0 \times 5 \mathrm{~A}$ & $0 \times 5 A$ & $0 \times 5 \mathrm{~A}$ \\
\hline $0 \mathrm{xCC}$ & $0 \mathrm{xCC}$ & $0 \mathrm{xCC}$ \\
\hline $0 x \mathrm{x} 8$ & $0 \mathrm{xD} 8$ & $0 \mathrm{xD} 8$ \\
\hline
\end{tabular}

\subsection{Measurement results}

In the echo test, one data byte is written to the microcontroller before reading the same data byte through the NFC-to-I2C interface. The data byte is randomly generated by the Android application which is controlling the NFC-to-I2C interface. The Android application then reports the transmitted data and the received data as hexadecimal values.

Furthermore, the microcontroller reports the value of the data byte to the host computer via the USB connection. The test was repeated 10 times, and the result of each test was evaluated. The results of the data reported by the Android application and the microcontroller controller (MCU) are shown in table 2. As seen in the table, the system passed all tests which means that the data transmission was successful in both directions.

In the second test, the task was to read the sensor information by using the NFC-to-I2C interface on the one hand and the microcontroller on the other. In order to be able to compare the results, we programmed the microcontroller to read the sensor 1.5 $\mathrm{s}$ after a specific request was sent from the smartphone (through the NFC-to-I2C interface). We chose $1.5 \mathrm{~s}$, because Sensirion recommends a delay of at least one second between each time the sensor is read.

In the test scenario, the smartphone reads the sensor, and then requests the microcontroller to read the sensor after a delay of $1.5 \mathrm{~s}$. This test was also repeated 10 times, with random delays of much more than $10 \mathrm{~s}$. Tables 3 and 4 present the obtained values for the temperature and relative humidity, respectively. Only minor differences can be noted in the
Table 3: Temperature readings by the smartphone through the NFC-to-I2C interface and by the microcontroller. The raw data in hexadecimal are listed in parenthesis.

\begin{tabular}{|c|c|}
\hline \multicolumn{2}{|c|}{ Temperature readings } \\
\hline NFC-I2C interface & \\
\hline $21.59^{\circ} \mathrm{C}(0 \times 63 \mathrm{~B} 4)$ & $\left.21.60^{\circ} \mathrm{C}(0 \times 63 \mathrm{~B} 8)\right)$ \\
\hline $21.59^{\circ} \mathrm{C}(0 \times 63 \mathrm{~B} 4)$ & $21.59^{\circ} \mathrm{C}(0 \times 63 \mathrm{~B} 4)$ \\
\hline $21.59^{\circ} \mathrm{C}(0 \times 63 \mathrm{~B} 4)$ & $21.59^{\circ} \mathrm{C}(0 \times 63 \mathrm{~B} 4)$ \\
\hline $21.58^{\circ} \mathrm{C}(0 \times 63 \mathrm{~B} 0)$ & $21.58^{\circ} \mathrm{C}(0 \times 63 \mathrm{~B} 0)$ \\
\hline $21.58^{\circ} \mathrm{C}(0 \times 63 \mathrm{~B} 0)$ & $21.58^{\circ} \mathrm{C}(0 \times 63 \mathrm{~B} 0)$ \\
\hline $21.58^{\circ} \mathrm{C}(0 \times 63 \mathrm{~B} 0)$ & $21.59^{\circ} \mathrm{C}(0 \mathrm{x} 63 \mathrm{~B} 4)$ \\
\hline $21.58^{\circ} \mathrm{C}$ & $21.59^{\circ} \mathrm{C}(0 \mathrm{x} 63 \mathrm{~B} 4)$ \\
\hline $21.58^{\circ} \mathrm{C}(0 \times 63 \mathrm{~B} 0)$ & $21.58^{\circ} \mathrm{C}(0 \mathrm{x} 63 \mathrm{~B} 0)$ \\
\hline $21.57^{\circ} \mathrm{C}(0 \times 63 \mathrm{AC})$ & $21.57^{\circ} \mathrm{C}(0 \times 63 \mathrm{AC})$ \\
\hline $21.57^{\circ} \mathrm{C}(0 \times 63 \mathrm{AC})$ & $21.58^{\circ} \mathrm{C}(0 \times 63 \mathrm{~B} 0)$ \\
\hline
\end{tabular}

Table 4: Relative humidity readings by the smartphone through the NFC-to-I2C interface and by the microcontroller. The raw data in hexadecimal are listed in parenthesis.

\begin{tabular}{cc}
\hline \multicolumn{3}{c}{ Relatice humidity readings } \\
NFC-I2C interface & Microcontroller \\
\hline $27.47 \% \mathrm{RH}(0 \mathrm{x} 448 \mathrm{E})$ & $27.47 \% \mathrm{RH}(0 \mathrm{x} 448 \mathrm{E})$ \\
$27.71 \% \mathrm{RH}(0 \mathrm{x} 450 \mathrm{~A})$ & $27.94 \% \mathrm{RH}(0 \mathrm{x} 4586)$ \\
$27.31 \% \mathrm{RH}(0 \mathrm{x} 443 \mathrm{~A})$ & $27.27 \% \mathrm{RH}(0 \mathrm{x} 4426)$ \\
$27.51 \% \mathrm{RH}(0 \mathrm{x} 44 \mathrm{~A} 2)$ & $27.51 \% \mathrm{RH}(0 \mathrm{x} 44 \mathrm{~A} 2)$ \\
$27.47 \% \mathrm{RH}(0 \mathrm{x} 448 \mathrm{E})$ & $27.51 \% \mathrm{RH}(0 \mathrm{x} 44 \mathrm{~A} 2)$ \\
$27.51 \% \mathrm{RH}(0 \mathrm{x} 44 \mathrm{~A} 2)$ & $27.67 \% \mathrm{RH}(0 \mathrm{x} 44 \mathrm{~F} 6)$ \\
$27.87 \% \mathrm{RH}(0 \mathrm{x} 455 \mathrm{E})$ & $27.82 \% \mathrm{RH}(0 \mathrm{x} 4546)$ \\
$27.74 \% \mathrm{RH}(0 \mathrm{x} 451 \mathrm{E})$ & $27.74 \% \mathrm{RH}(0 \mathrm{x} 451 \mathrm{E})$ \\
$27.47 \% \mathrm{RH}(0 \mathrm{x} 448 \mathrm{E})$ & $27.67 \% \mathrm{RH}(0 \mathrm{x} 44 \mathrm{~F} 6)$ \\
$27.47 \% \mathrm{RH}(0 \mathrm{x} 448 \mathrm{E})$ & $27.67 \% \mathrm{RH}(0 \mathrm{x} 44 \mathrm{~F} 6)$ \\
\hline
\end{tabular}

obtained results which are present due to noise and small fluctuations in ambient conditions. The range of measured values is $21.58{ }^{\circ} \mathrm{C} \pm 0.01^{\circ} \mathrm{C}$ and $27.6 \% R H \pm 0.4 \% R H$ measured by the smartphone through the NFC-to-I2C interface, and $21.58^{\circ} \mathrm{C} \pm$ $0.02^{\circ} \mathrm{C}$ and $27.6 \% R H \pm 0.4 \% R H$ obtained with help of the microcontroller.

Since the measurement results of the NFC-to-I2C interface and the microcontroller are nearly identical, it can be stated that the type of interface does not have an influence on measurement results. Furthermore, the uncertainties in the readings of the sensor $\left( \pm 0.3^{\circ} \mathrm{C}\right.$ and $\pm 2 \% \mathrm{RH}$ in the datasheet [25]) are significantly 
higher than the difference in the obtained measurement results.

\subsection{Practical application of the proposed system}

Nowadays, smartphones contain a remarkable amount of embedded sensors. For example, the Samsung Galaxy S7 Edge features sensors which allow users measuring their heart rate and the oxygen saturation degree of arterial blood (SpO2). Data obtained by these types of sensors is then used by mobile applications such as Samsung S Health to provide users valuable information of their health and well-being.

Recently, light sensors available in smartphones were used to estimate the amount of daily illumination in order to investigate the circadian rhythm and influences on the sleep-wake cycle of humans [26]. However, even though the amount of sensors of smartphones has increased over time significantly, yet, not all types of sensors are available on mobile phones.

In addition, significant errors can be present in the readings of integrated smartphone sensors such as the ambient light sensor (ALS). A calibration can be required to lower measurement errors [2]. Generally speaking, it can be helpful and beneficial for smartphone users to obtain data from external sensors such as ambient light sensors and temperature sensors, for example.

In our proposed system, external sensor data can be read in a simple way via NFC with the help of the smartphone. In this way, information on the conditions of the surrounding environment can be obtained with any type of smartphone which includes NFC. As a result, the smartphone can act as a measurement instrument and collected data can be used by various different types of mobile applications directly.

For example, the Vernier Sensor data acquisition module (DAQ) allows interfacing with over 60 different types of analog and digital sensors. In a similar way, our interface can be used to allow smartphones communicating with a large number of different types of external sensors via NFC.

In this part of our research work, the focus lies again on temperature sensors. At the moment, mobile applications are available which display information from different sensors in smartphones free-of-charge. These applications are helpful to display the current value, for example the light intensity (in lux) measured with the help of the ALS.

Figure 9 shows one of the typical applications which can be found in Google Play. It is worth noting that only the Samsung Galaxy S4 features a temperature/humidity sensor, while successors of the S4 such as the S5, S6, and S7 do not contain an ambient temperature sensor. Similarly, the predecessors such as the S2 and S3 do not have a temperature/humidity

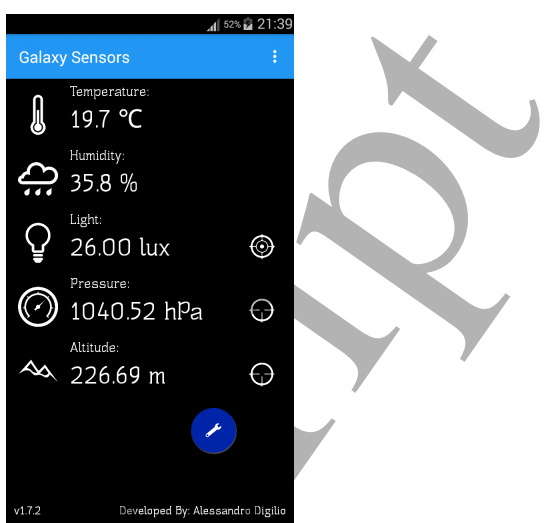

Figure 9: Example for a mobile application on a Samsung Galaxy S3 for obtaining sensor information.

sensor. However, if the application is installed, for example on a Samsung Galaxy S3, information on the available temperature is presented to the user.

It is worth noting that the displayed temperature is actually not the ambient temperature. Instead, the smartphones' battery temperature is displayed by the application. The question is how suitable the smartphones' battery temperature is for providing information on the ambient temperature. In our experiments, we used two Android smartphones, a Samsung Galaxy S3 (1) (GT-I9305) and a Samsung Galaxy S3 (2) (SCH-L710) as well as a conventional temperature meter from Voltcraft DT-300 (tolerance $\pm 1{ }^{\circ} \mathrm{C}$ by the manufacturer).

The Voltcraft DT-300 temperature meter was used as reference in the experiments. We built a test environment, shown in figure 10, for comparing the results of temperature readings between Android smartphones ( $\left.T_{\text {phone }}\right)$ and a conventional temperature meter $\left(T_{r e f}\right)$. Here, the temperature meter was placed as close as possible to the smartphone. The test environment allowed us to control the temperature increase inside. A digital temperature sensor (Maxim DS18B20) connected to a microcontroller was used as additional reference as well as for controlling the temperature inside the test environment. Experiments were repeated several times in order to conduct a statistical evaluation of the measurement results.

Each time, the test started at a room temperature of $22{ }^{\circ} \mathrm{C}$. Then, the temperature was increased until 36 ${ }^{\circ} \mathrm{C}$ were reached. Here, the temperature was increased with a rate $\left(\frac{d T}{d t}\right)$ of about $0.025 \frac{{ }^{\circ} C}{s}$ or $1.5 \frac{{ }^{\circ} C}{\min }$. Afterwards, a built-in ventilation system controlled by a microcontroller was used to cool the test environment down until the room temperature was reached again. Similarly, the smartphones were let to rest and to cool down until they reached the initial temperature level again.

The temperature was monitored on a continuous 


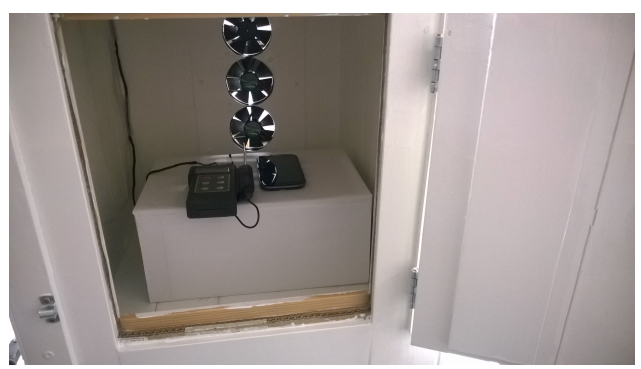

Figure 10: Test environment for measuring temperatures with Android smartphones and a conventional temperature meter.

basis throughout the whole experiment. For an easier comparison of the measurement results, we show an evaluation every $2{ }^{\circ} \mathrm{C}$. Figure 11 shows the temperature readings of the Samsung Galaxy S3 (1) (GT-I9305), while figure 12 shows the readings with the help of the Samsung Galaxy S3 (2) (SCH-L710).

As seen in figure 11, already in the beginning of the experiment, the smartphone obtained a lower temperature $\left(T_{\text {phone }}\right)$ of about $4{ }^{\circ} \mathrm{C}$ compared to the Voltcraft DT-300 temperature meter $\left(T_{\text {ref }}\right)$. This obtained temperature difference increased during the experiment. At $T_{r e f}=36{ }^{\circ} \mathrm{C}$, the difference in measurement results was about $8{ }^{\circ} \mathrm{C}$. A similar situation can be observed in the measurements with the second smartphone in figure 12.

In addition, the spread in sensor readings (indicated as error bars in figures 11 and 12) increased when the temperature was increased in the test environment. Moreover, the dynamic response of the smartphones' sensor was poor. The Samsung Galaxy S3 (1) (GT-I9305), for example, did not immediately respond to temperature changes while the reference temperature meter measured temperature changes with a step of $0.1^{\circ} \mathrm{C}$.

In summary, the temperature readings obtained with smartphones do not provide accurate information on the ambient temperature. Thus, we conducted the same experiment with our proposed system and the Sensirion SHT21 humidity and temperature sensor. The temperature information from the sensor $\left(T_{N F C}\right)$ was read through NFC with the help of one of the Samsung smartphones.

Figure 13 shows the obtained measurement results. It can be seen that the temperature readings with the sensor from Sensirion $\left(T_{N F C}\right)$ were very similar with the results of the reference temperature meter from Voltcraft $\left(T_{\text {ref }}\right)$ throughout the experiment. The Sensirion SHT21 humidity and temperature sensor showed a prompt response. Each time temperature information was requested, the current temperature value was available immediately. Furthermore, the

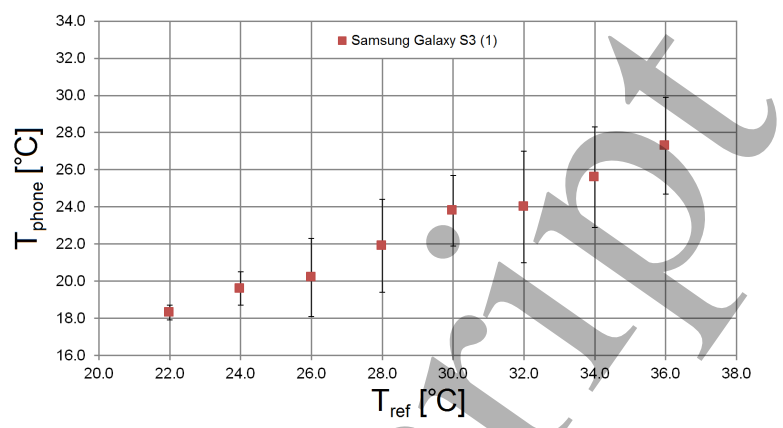

Figure 11: Obtained temperature readings at reference points with the Samsung Galaxy S3 (1) (GT-I9305).

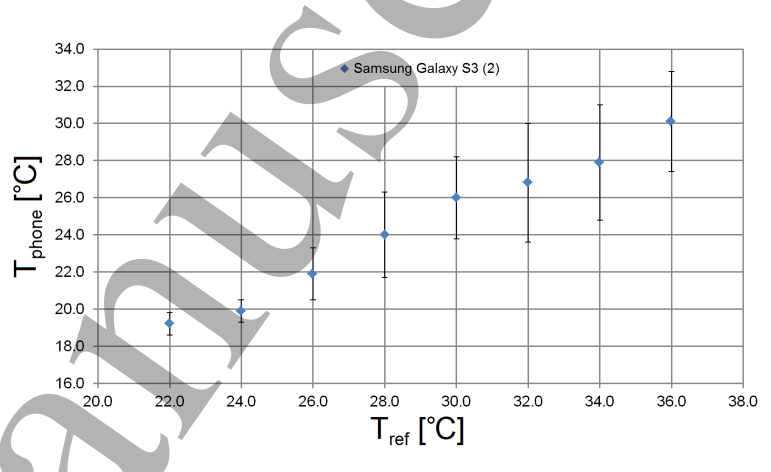

Figure 12: Obtained temperature readings at reference points with the Samsung Galaxy S3 (2) (SCH-L710).

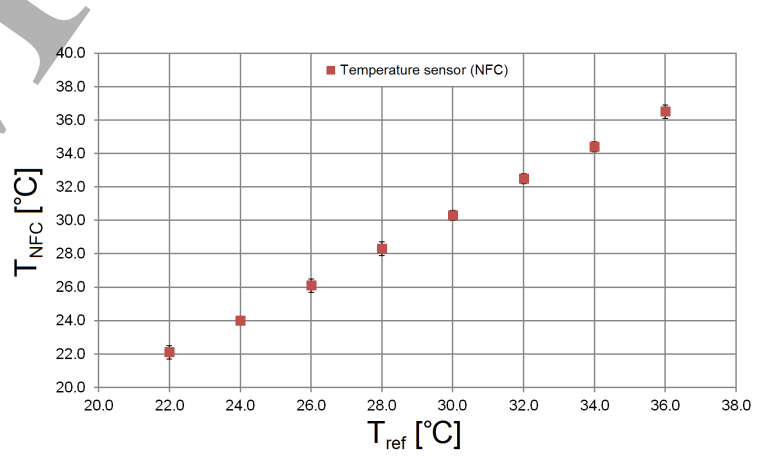

Figure 13: Obtained temperature readings at reference points with the Sensirion SHT21 humidity and temperature sensor read via NFC with a smartphone.

measurement results were very consistent and no significant differences in sensor readings were obtained when the experiment was repeated (indicated as error bar in figures 13).

\section{Discussion and Conclusion}

In this paper, we presented a new approach for enabling a communication with sensors and other I2C devices with NFC. Our proposed system has been successfully tested and evaluated with the help of smartphones which feature NFC. Our approach eliminates the need of programming microcontrollers on the sensor side of 
the system, by implementing an I2C master core in the NFC circuit which can be controlled by a simple non-standard NFC command.

As a result, only the mobile application on the Android smartphone needs to be programed, which makes the use of the NFC circuit very flexible and easy to work with in practice. In addition, our approach reduces the delay in data transfer from the smartphone to an $\mathrm{I} 2 \mathrm{C}$ chip, because the NFC circuit no longer needs to be polled from both sides for the data transmission.

We elaborated the digital design of our system. In experiments, it was shown that the transmitted data and received data through the NFC-to-I2C interface indeed is valid. We conducted our tests with a commercially available humidity and temperature sensor, more precisely the Sensirion SHT21, as well as a microcontroller featuring a hardware $\mathrm{I} 2 \mathrm{C}$ port, the Atmel Atmega328P.

Nowadays, the large majority of the Android smartphones features NFC. However, Android smartphones do not feature certain types of sensors such as a temperature/humidity sensor. Generally speaking, sensor data can be helpful especially for improving the living quality and health of humans, for example in indoor environments.

In mobile applications which are available free-ofcharge from the application store, the smartphones? battery temperature is read instead of the ambient temperature. Thus, users of smartphones can obtain wrong sensor information about their surrounding environment. We built a test environment to analyze the capability of our system to obtain sensor data from external temperature sensors via NFC.

It was shown that the proposed system provides accurate measurement data instantaneously. Moreover, temperature readings were very consistent with the readings of a conventional temperature meter, the Voltcraft DT-300 which we used in our experiments. Our system can be beneficial to interface various different types of external sensors in a simple way with smartphones via NFC.
[1] "About Near http://www.nearfieldcommunication.org/aboutnfc.html. Accessed 04.03.2016.

[2] "Ways to Use Near Field Communications", http://www.nearfieldcommunication.org/usingnfc.html. Accessed 04.03.2016.

[3] J. Maxa, T. Krachenfels and H. Beikirich, "Near Field Communication Interface for a Packet-Based Serial Data Transmission Using a Dual Interface EEPROM", Emerging Technologies \& Factory Automation (ETFA), 2015 IEEE 20th Conference on, 2015, pp. 1-4.

[4] "NTAG I2C - Energy harvesting NFC Forum Type 2 Tag with field detection pin and $\mathrm{I} 2 \mathrm{C}$ interface datasheet NT3H1101/NT3H1201", technical report, 2015.

[5] M. Hillukkala, M. Heiskanen and A. Ylisaukko-oja, "Practical implementations of passive and semi-passive NFC enabled sensors", Near Field Communication, 2009 First International Workshop on, 2009.
[6] R. Trevisan and A. Costanzo, A., "Exploitation of passive RFID technology for wireless read-out of temperature sensors", IEEE RFID Technology and Applications Conference (RFID-TA), 2014, pp. 150-154.

[7] J. Haase, D. Meyer, M. Eckert, and B. Klauer, "Wireless sensor/actuator device configuration by NFC", IEEE International Conference on Industrial Technology (ICIT), pp. 1336-1340, 2016.

[8] A. DeHennis, S. Getzlaff, D. Grice, and M. Mailand, "An NFC-Enabled CMOS IC for a Wireless Fully Implantable Glucose Sensor", IEEE journal of biomedical and health informatics, vol. 20, issue 1, pp. 18-28, 2016.

[9] N. Anabtawi, S. Freeman, and R. Ferzli, "A fully implantable, NFC enabled, continuous interstitial glucose monitor", IEEE-EMBS International Conference on Biomedical and Health Informatics (BHI), pp. 612-615, 2016.

[10] A. Zaher, J. Saersten, T.T. Nguyen, and P. Hfliger, "Integrated electronic system for implantable sensory NFC tag", 37th Annual International Conference of the IEEE Engineering in Medicine and Biology Society (EMBC), pp. 7119-7122, 2015.

[11] J. Vanwalleghem, I. De Baere, M. Loccufier, and W. Van Paepegem, "Sensor design for outdoor racing bicycle field testing for human vibration comfort evaluation, Measurement Science and Technology, vol. 24, issue 9, pp. 095002, 2013.

[12] X. Guo, Y. Huang, X. Cai, C. Liu, and P. Liu, "Capacitive wearable tactile sensor based on smart textile substrate with carbon black/silicone rubber composite dielectric, Measurement Science and Technology, vol. 27, issue 4, pp. 045105, 2016.

[13] P. Lv, Z. Tang, G. Wei, J. Yu, and Z. Huang, "Recognizing indoor formaldehyde in binary gas mixtures with a micro gas sensor array and a neural network. Measurement Science and Technology, vol. 18, issue 9, pp. 2997, 2007.

[14] A. P. Jones, "Indoor air quality and health", Atmospheric environment, vol. 33, issue 28, pp. 4535-4564, 1999.

[15] I. Turiel, "Indoor air quality and human health", Routledge, 1998.

[16] A. Szczurek, and M. Maciejewska, "Gas sensing method applicable to real conditions", Measurement Science and Technology, vol. 24, issue 4, pp. 045103, 2013.

[17] P. Wide, F. Winquist, and D. Driankov, "An air-quality sensor system with fuzzy classification, Measurement Science and Technology, vol. 8, issue 2, pp. 138, 1997.

[18] S. Ahson and M. Ilyas, "RFID handbook : applications, technology, security, and privacy", Boca Raton: CRC Press, 2008.

[19] P. Corcoran, "Two Wires and 30 Years : A Tribute and Introductory Tutorial to the I2C Two-Wire Bus",Consumer Electronics Magazine, IEEE, vol. 1, issue 3, pp. 30-36, 2013.

[20] "Type 2 Tag Operation", NFC Forum Technical Specification, Rev. 1.2, 2014.

[21] "NFC Digital Protocol", NFC Forum Technical Specification, Rev. 1.1, 2014.

[22] "I2C-bus specification and user manual", NXP Semiconductors, Rev. 6, 2014.

[23] "The Chameleon project", https://github.com/emsec/ ChameleonMini/wiki. Accessed 26.02.2016.

[24] "Arduino/Genuino UNO", https://www.arduino.cc/ en/Main/ArduinoBoardUno. Accessed 26.02.2016.

[25] "Datasheet SHT21. Humidity and Temperature Sensor IC", technical report, 2014.

[26] F. Wahl, T. Kantermann, and O. Amft "How much light do you get?: estimating daily light exposure using smartphones", Proceedings of the 2014 ACM International Symposium on Wearable Computers, pp. 43-46, 2014. 